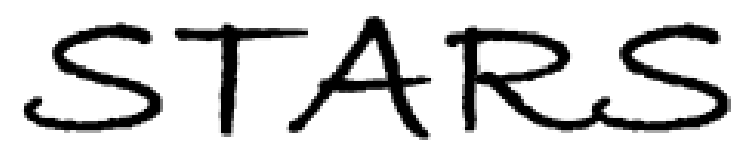

University of Central Florida

STARS

$1-1-1997$

\title{
Transform coding of signals using approximate trigonometric expansions
}

Qurban A. Memon

University of Central Florida

Takis Kasparis

University of Central Florida

Find similar works at: https://stars.library.ucf.edu/facultybib1990

University of Central Florida Libraries http://library.ucf.edu

This Article is brought to you for free and open access by the Faculty Bibliography at STARS. It has been accepted for inclusion in Faculty Bibliography 1990s by an authorized administrator of STARS. For more information, please contact STARS@ucf.edu.

\section{Recommended Citation}

Memon, Qurban A. and Kasparis, Takis, "Transform coding of signals using approximate trigonometric expansions" (1997). Faculty Bibliography 1990s. 2013.

https://stars.library.ucf.edu/facultybib1990/2013

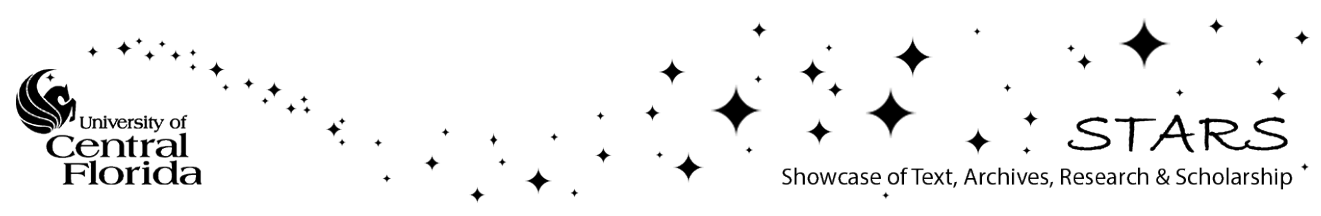




\title{
Transform coding of signals using approximate trigonometric expansions
}

\author{
Qurban A. Memon* \\ Takis Kasparis \\ University of Central Florida \\ Department of Electrical and Computer Engineering \\ P.O. Box 162450 \\ Orlando, Florida 32816 \\ E-mail: tnk@engr.ucf.edu
}

\begin{abstract}
For signal representation, it is always preferred that a signal be represented using a minimum number of parameters. In any transform coding scheme, the central operation is the reduction of correlation and thereby, with appropriate coding of the transform coefficients, allows data compression to be achieved. The objective of data encoding is to transform a data array into a statistically uncorrelated set. This step is typically considered a "decorrelation" step, because in the case of unitary transformations, the resulting transform coefficients are relatively uncorrelated. Most unitary transforms have the tendency to compact the signal energy into relatively few coefficients. The compaction of energy thus achieved permits a prioritization of the spectral coefficients, with the most energetic ones receiving a greater allocation of encoding bits. The transform efficiency and ease of implementation are to a large extent mutually incompatible. There are various transforms such as KarhunenLoeve, discrete cosine transforms, etc., but the choice depends on the amount of reconstruction error that can be tolerated and the computational resources available. We apply an approximate Fourier series expansion (AFE) to sampled one-dimensional signals and images, and investigate some mathematical properties. Additionally, we extend the expansion to an approximate cosine expansion (ACE) and show that, for the purpose of data compression with minimum error reconstruction of images, the performance of ACE is better than AFE. For comparison purposes, the results are also compared with a discrete cosine transform (DCT). (C) 1997 SPIE and IS\&T. [S1017-9909(97)01004-0]
\end{abstract}

\section{Introduction}

With the continuing growth of modern communications technology, demand for data storage and transmission is rapidly increasing. The efficiency, complexity, as well as implementation of a compression algorithm are particularly important in its hardware implementation. Transform coding is one of the well-known approaches to efficient waveform representation at medium to low bit rates. The goal of transform coding is to decorrelate the signal, resulting in the energy being distributed among only a small set of coefficients. In this way, many coefficients can be discarded

Paper 96-026 received May 1996; accepted for publication March 1997. 1017-9909/97/\$10.00 @ 1997 SPIE and IS\&T.

*Current affiliation: GIK Institute of Engineering and Technology, Topi Swabi, Pakistan. after quantization and prior to encoding. A transformation can be viewed as a decomposition of the original block of signals into a set of basis functions. In the case of sinusoidal transforms (such as Fourier transform), the basis functions consist of sines and/or cosines with different spatial frequencies, and each transform coefficient is proportional to the fraction of energy in the original block at that particular frequency. It is important to realize that the transform operation by itself does not achieve any compression, but by changing the representation of the information contained in the signal block, it makes the data more suitable for compression. Compression is achieved by subsequent steps of quantization and encoding of the transform coefficients. The optimal decorrelation transformation is the Karhunen-Loeve transform (KLT). ${ }^{1}$ The KLT has the property that for any integer $L \leqslant N$, where $L$ is the size of the transform and $N$ is the size of the data vector, it packs the maximum average energy into some $L$ coefficients. ${ }^{2}$ Unfortunately, no efficient computation of KLT exists, and also it does not have the desirable properties of a trigonometric series. Another complication in applying KLT is that its basis functions are not fixed but are data dependent. Inputdependent transforms are hard to implement, but they also have the best input decorrelating and variance ordering properties. A practical transform for the purpose of signal compression should have a strong decorrelating effect, should preferably consist of signal-independent basis functions, and should have a fast implementation. For stationary random sequences there are other unitary transforms which approach the energy packing efficiency of the KLT. Examples are discrete cosine, Fourier, and sine transforms. These transforms are members of a large family of sinusoidal transforms, all of which have a performance equivalent to KLT as the size $N$ of the data vector approaches infinity. ${ }^{3}$ To retain the desirable features of the trigonometric series, an approximate Fourier expansion with uncorrelated coefficients for continuous-time nonperiodic signals, can be found in Ref. 4. The approximation can be made arbitrarily close by making a transform parameter small enough. In this paper, we apply an approximate Fourier expansion (AFE) to sampled signals and explore the capa- 
bility of coding one-dimensional signals and images. Furthermore, we extend this expansion to an approximate cosine expansion (ACE) and show that, for the purpose of coding and minimum error reconstruction of signals, its performance is better than AFE. Performance is also compared with the discrete cosine transform.

\section{Approximate Fourier Expansion}

In this section, we present an AFE of sampled, stationary, and nonperiodic signals. For continuous-time signals, this expansion is expressed as ${ }^{4}$ :

$\hat{x}(t)=\sum_{k=-\infty}^{\infty} c_{k} \exp \left(j k \omega_{0} t\right)$,

where $c_{k}$ are random variables given by

$c_{k}=\int_{-\infty}^{\infty} x(t) \frac{\sin \left(\frac{\omega_{0} t}{2}\right)}{\pi t} \exp \left(-j k \omega_{0} t\right) d t$.

For sampled signals, we extend the approximate Fourier expansion as

$\hat{x}(n)=\sum_{k=-M}^{M} c_{k} \exp \left(j k \omega_{0} n\right)$,

where

$$
\begin{gathered}
c_{k}=\sum_{n=-\infty}^{\infty} x(n) \frac{\sin \left(\frac{\omega_{0} n}{2}\right)}{\pi n} \exp \left(-j k \omega_{0} n\right), \\
k=0, \pm 1, \ldots, \pm M,
\end{gathered}
$$

where $M$ is the maximum number of coefficients to be computed. If we divide the frequency spectrum $2 \pi$ by frequency resolution parameter $\omega_{0}$, then we have a total number $L$ of coefficients. Hence, for signals of finite length $N$, we can write discrete AFE as

$c_{k}=\sum_{n=0}^{N-1} x(n) \frac{\sin \left(\frac{\pi n}{L}\right)}{\pi n} \exp \left(-j \frac{2 \pi k n}{L}\right), \quad k=0,1, \ldots L-1$.

We show later that, depending on the value of $\omega_{0}$ $=2 \pi / L$, the coefficients $c_{k}$ could be periodic or pseudoperiodic. The signal $x(n)$ can be reconstructed as

$\hat{x}(n)=\sum_{k=0}^{L-1} c_{k} \exp \left(j \frac{2 \pi k n}{L}\right)$.

If the signal $x(n)$ is of finite duration $N=L$, then the pair of Eqs. (3) and (4) can be viewed as a discrete Fourier transform (DFT) pair of the signal $x(n)[\sin (\pi n / L) / \pi n]$, i.e., the signal $x(n)$ windowed by the main lobe of the sinc function.

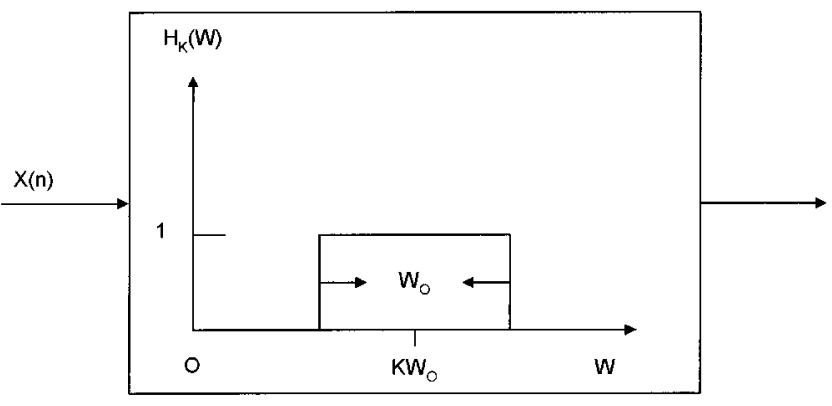

Fig. 1 Ideal bandpass filter.

\section{Properties of the Expansion}

In this section, we examine some important properties of this expansion. For now, we consider only the case of onedimensional sampled signals.

\subsection{Mean Value of Coefficients}

Property.

$E\left\{c_{k}\right\}=\left\{\begin{array}{ll}E\{x(n)\} & \text { for } k=0 \\ 0 & \text { otherwise }\end{array}\right.$.

Proof. From Equation (2b)

$E\left\{c_{k}\right\}=E\{x(n)\} \sum_{n=-\infty}^{\infty} \frac{\sin \left(\omega_{0} n / 2\right)}{\pi n} \exp \left(-j k \omega_{0} n\right)$.

The summation term in this equation represents the Fourier transform of $\sin \left(\omega_{0} n / 2\right) / \pi n$ evaluated at $\omega=k \omega_{0}$. Since the Fourier transform of $\sin \left(\omega_{0} n / 2\right) / \pi n$ is bandlimited between $-\omega_{0} / 2$ and $\omega_{0} / 2$, hence the right-hand side of Equation (6) is zero, except when $k=0$ where it has the value of 1 , the property is proved.

\subsection{Correlation of Coefficients}

Property. The coefficients of the AFE are uncorrelated.

Proof. Consider the ideal bandpass filters of center frequency $k \omega_{0}$ and bandwidth $\omega_{0}$ as in Fig. 1:

$H_{k}(\omega)=\left\{\begin{array}{ll}1 & \left(k-\frac{1}{2}\right) \omega_{0}<\omega<\left(k+\frac{1}{2}\right) \omega_{0} \\ 0 & \text { otherwise }\end{array}\right.$,

where $-\pi \leqslant \omega \leqslant \pi$ is a frequency variable. The corresponding impulse response is given by

$h_{k}(n)=\exp \left(j k \omega_{0} n\right) \frac{\sin \left(\frac{\omega_{0} n}{2}\right)}{\pi n}$.

If $x(n)$ is the input to this filter, the output of the $k$ 'th bandpass filter will be given by the convolution summation

$y_{k}(n)=\sum_{\tau=-\infty}^{\infty} x(\tau) \exp \left[j k \omega_{0}(n-\tau)\right] \frac{\sin \left[\frac{\omega_{0}(n-\tau)}{2}\right]}{\pi(n-\tau)}$.

At $n=0$ we have 
$y_{k}(0)=\sum_{\tau=-\infty}^{\infty} x(\tau) \exp \left(-j k \omega_{0} \tau\right) \frac{\sin \left(\frac{\omega_{0} \tau}{2}\right)}{\pi \tau}=c_{k}$.

Since individual filters are nonoverlapping, their outputs $c_{k}$ and $c_{m}$ are orthogonal, i.e.,

$E\left\{y_{k}(0) y_{m}^{*}(0)\right\}=E\left\{c_{k} c_{m}^{*}\right\}=0$, for $k \neq m$.

Since from the property in Sec. 3.1, $E\left\{c_{k}\right\}=0$ except at $k$ $=0$, we conclude that the coefficients are also uncorrelated.

\subsection{Periodicity}

Property. If $\omega_{0}=2 \pi / L$, and $L$ is a rational number, then the coefficients $c_{k}$ and the reconstructed signal $\hat{x}(n)$ will be periodic.

Proof. From (3) it is evident that if the complex exponential is periodic, then the coefficients will be periodic in $k$. If $L=P / Q$ where $P, Q$ are integers and $L$ is a rational number, then $\exp (-j 2 \pi Q / P k n)$ will be periodic with period $P$. From (4) it can be noticed that the reconstructed signal will be also periodic with the same period. Under these conditions, the upper limit of the summation in (4) can be $P$. In the special case where $L$ is an integer $(Q=1)$, then the period will be $L$. It should also be noted that when $L$ is an irrational number, then there will be a "pseudoperiodicity" in the coefficients with period $P=\operatorname{int}\{L\}$, i.e., the coefficients within a pseudoperiod $P$ will not be equal but approximately similar. From this it can be concluded that an integer value of $L$ will always result in a computation of less number of coefficients.

\subsection{Mean Value of Reconstructed Signal}

Property.

$E\{\hat{x}(n)\}=E\{x(n)\}$.

Proof. Using Equation (4),

$E\{\hat{x}(n)\}=E\left[\sum_{k=0}^{L-1} c_{k} \exp \left(j \frac{2 \pi k n}{L}\right)\right]$.

Using the property in Sec. 3.1,

$E\left\{c_{k}\right\}=\left\{\begin{array}{ll}E\{x(n)\} & \text { for } k=0 \\ 0 & \text { otherwise }\end{array}\right.$.

At $k=0$, and using this result, we have in mean square sense

$E\{\hat{x}(n)\}=E\left\{c_{0}\right\}=E\{x(n)\}$.

This equation suggests that reconstructed signal $\hat{x}(n)$ gives mean square approximation to $x(n)$.

\subsection{Mean Square Value of Coefficients}

Property.

$E\left\{\left|c_{k}\right|^{2}\right\}=\frac{1}{2 \pi} \int_{(k-1 / 2) \omega_{0}}^{(k+1 / 2) \omega_{0}} S_{x}(\omega) d \omega$.
Proof. Consider the ideal bandpass filters of center frequency $k \omega_{0}$ and bandwidth $\omega_{0}$ as in Fig. 1:

$H_{k}(\omega)= \begin{cases}1 & \left(k-\frac{1}{2}\right) \omega_{0}<\omega<\left(k+\frac{1}{2}\right) \omega_{0}, \\ 0 & \text { otherwise }\end{cases}$

where $-\pi \leqslant \omega \leqslant \pi$ is a frequency variable. by

The power spectrum of output of the subfilter $k$ is given

$S_{y}(\omega)=S_{x}(\omega)\left|H_{k}(\omega)\right|^{2}$.

Taking inverse Fourier transform of both sides, we get

$E\left\{\left|y_{k}(n)\right|^{2}\right\}=\frac{1}{2 \pi} \int_{-\pi}^{\pi} S_{x}(\omega)\left|H_{k}(\omega)\right|^{2} \exp (j \omega n) d \omega$.

Using the limits of $H_{k}(\omega)$, we get at $n=0$

$E\left\{\left|y_{k}(0)\right|^{2}\right\}=\frac{1}{2 \pi} \int_{(k-1 / 2) \omega_{0}}^{(k+1 / 2) \omega_{0}} S_{x}(\omega) d \omega$.

Using the result in Equation (10) we get

$E\left\{\left|c_{k}\right|^{2}\right\}=\frac{1}{2 \pi} \int_{(k-1 / 2) \omega_{0}}^{(k+1 / 2) \omega_{0}} S_{x}(\omega) d \omega$

which is the area under the $k$ 'th pulse. If $\omega_{0}$ is very small, so that $S_{x}(\omega)$ is a constant $S_{x}$ in that interval, then

$E\left\{\left|c_{k}\right|^{2}\right\}=\frac{S_{x}}{2 \pi} * \omega_{0}=\frac{S_{x}}{L}$,

which means that we are sampling the spectrum of the original signal at sufficient number $L$ of frequency points with an area of each impulse as $S_{x} / L$.

\subsection{Mean Square Value of Reconstructed Signal}

Property.

$E\left\{|\hat{x}(n)|^{2}\right\}=E\left\{|x(n)|^{2}\right\}$.

Proof. From Equation (4),

$$
\begin{aligned}
E\left\{|\hat{x}(n)|^{2}\right\}= & E\left\{\sum_{k=0}^{L-1} c_{k} \exp \left(j k \omega_{0} n\right) \sum_{m=0}^{L-1} c_{m}\right. \\
& \left.\times \exp \left(-j m \omega_{0} n\right)\right\} .
\end{aligned}
$$

Using $\omega_{0}=2 \pi / L$ with $L$ as an integer, we have

$$
\begin{aligned}
E\left\{|\hat{x}(n)|^{2}\right\} & =E\left\{\sum_{k=0}^{L-1} \sum_{m=0}^{L-1} c_{k} c_{m} \exp \left[j(k-m) \omega_{0} n\right]\right\} \\
& =\sum_{k=0}^{L-1} \sum_{m=0}^{L-1} E\left\{c_{k} c_{m}\right\} E\left\{\exp \left[j \frac{2 x(k-m) n}{L}\right]\right\},
\end{aligned}
$$




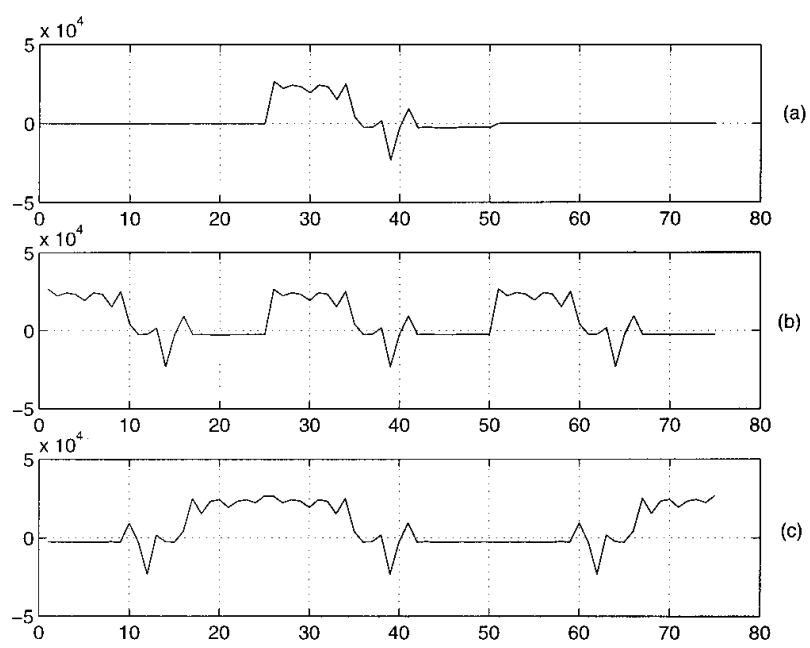

Fig. 2 (a) Signal length for analysis, (b) data domain equivalent of AFE coefficients, and (c) data folded to eliminate discontinuity.

$$
\begin{aligned}
E\left\{|\hat{x}(n)|^{2}\right\}= & \sum_{k=0}^{L-1} \sum_{m=0}^{L-1} E\left\{c_{k} c_{m}\right\} * \frac{1}{N} \\
& \times \sum_{n=0}^{N-1} \exp \left[j \frac{2 \pi(k-m) n}{L}\right] .
\end{aligned}
$$

Using the result in Equation (11), we have at $k=m$

$E\left\{|\hat{x}(n)|^{2}\right\}=\sum_{k=0}^{L-1} E\left\{\left|c_{k}\right|^{2}\right\}$.

Using the property in Sec. 3.5 we get

$E\left\{|\hat{x}(n)|^{2}\right\}=\sum_{k=0}^{L-1} \frac{1}{2 \pi} \int_{(k-1 / 2) \omega_{0}}^{(k+1 / 2) \omega_{0}} S_{x}(\omega) d \omega$.

If $\omega_{0}$ is very small, so that $L=2 \pi / \omega_{0}$ is sufficiently large for minimum reconstruction error and $S_{x}(\omega)$ is constant in that interval $\omega_{0}$, then the previous equation becomes

$E\left\{|\hat{x}(n)|^{2}\right\}=\frac{1}{2 \pi} \int_{-\pi}^{\pi} S_{x}(\omega) d \omega$,

$E\left\{|\hat{x}(n)|^{2}\right\}=E\left\{|x(n)|^{2}\right\}$.

However, if $\omega_{0}$ is not very small, then there will be an error in the approximation of the original signal spectrum. In that case, the mean square value of the reconstructed signal will be given by Equation (26).

\section{Approximate Cosine Expansion}

In this section, we develop an ACE from the AFE and show that for the same $L$ it introduces less reconstruction error than the AFE. An additional advantage is that the coefficients of ACE are real. Consider Figs. 2(a) and 2(b), which represent sampled data and the reconstructed signal after applying an AFE. It should be noted that $L$ used was an integer and sufficiently large for minimum reconstruction error. Since the AFE coefficients and the reconstructed sig- nal are periodic with period $L$, Fig. 2(b) contains severe discontinuities between the segments and these result in spurious spectral components. This discontinuity can be removed by making the data to be transformed symmetric, i.e., by folding it about the vertical axis (along the origin) as shown in Fig. 2(c), and then overlapping the two halves by one element. Folding the data has given us an even function to transform. We now apply AFE of length $2 L$ to the data of length $2 N$. Note that the axis of symmetry in Fig. 2(c) lies at the point $n=-1 / 2$, i.e., $1 / 2$ point to the left of the signal at the origin. Therefore, applying AFE to the signal in Fig. 2(c), we get

$c_{k}=\sum_{n=-N}^{N-1} x(n) \frac{\sin \left(\frac{n \pi}{2 L}\right)}{n \pi} \exp \left[-j \frac{2 \pi k\left(n+\frac{1}{2}\right)}{2 L}\right]$.

Since $x(n)$ is real and even, therefore

$$
\begin{gathered}
c_{k}=2 \sum_{n=0}^{N-1} x(n) \frac{\sin \left(\frac{n \pi}{2 L}\right)}{n \pi} \cos \left[\frac{\pi k(2 n+1)}{2 L}\right], \\
k=0,1, \ldots, L-1 .
\end{gathered}
$$

The $x(n)$ is reconstructed using Equation (4) as

$$
\begin{aligned}
\hat{x}(n) & =\sum_{k=0}^{L-1} c_{k} \exp \left[j \frac{\pi k(2 n+1)}{2 L}\right] \\
& =\sum_{k=0}^{L-1} c_{k} \cos \frac{\pi k(2 n+1)}{2 L}+j \sum_{k=0}^{L-1} c_{k} \sin \frac{\pi k(2 n+1)}{2 L} .
\end{aligned}
$$

Since $c_{k}$ is real and even as it is generated by symmetric extension of input signal, hence the second term in the previous equation equates to zero, therefore

$\hat{x}(n)=\sum_{k=0}^{L-1} c_{k} \cos \frac{\pi k(2 n+1)}{2 L}$.

Letting $L_{1}=2 L$, then Equation (29) can be rewritten as

$$
\begin{aligned}
c_{k}= & 2 \operatorname{Re}\left[\sum_{n=0}^{N-1} x(n) \frac{\sin \left(\frac{n \pi}{L_{1}}\right)}{n \pi} \exp \left(-j \frac{2 \pi k n}{L_{1}}\right)\right. \\
& \left.\times \exp \left(-j \frac{\pi k}{L_{1}}\right)\right] .
\end{aligned}
$$

The term in the brackets is similar to $c_{k}$ in Equation (3) (with $L$ replaced by $L_{1}$ ) multiplied by an exponential term. Since $L_{1}=2 L$, therefore $\omega_{0}=2 \pi / L_{1}=\pi / L$. This means that first null of $\sin \left(n \pi / L_{1}\right)$ will fall at $2 L$ instead of at $L$. In other words, the error introduced in the reconstructed signal will be less than the error due to applying AFE on the signal. 


\section{Mean Square Error}

In this section, we compute the mean square error between the original and reconstructed signal. The main objective here is to evaluate an upper bound on $\omega_{0}$ so that error does not exceed a certain percentage of the average power of $x(n)$. We define the mean square error as

$e_{m s}=E\left\{|x(n)-\hat{x}(n)|^{2}\right\}$,

$e_{m s}=2 E\left\{|\hat{x}(n)|^{2}\right\}-2 E\left\{\left|x(n) \hat{x}(n)^{*}\right|\right\}$.

We have used the property in Sec. 3.6 that $E\left\{|x(n)|^{2}\right\}$ $=E\left\{|\hat{x}(n)|^{2}\right\}$. Using $c_{k}=y_{k}(0)$ (output of subfilter $k$ ) as shown in Equation (10), we get

$E\left\{x(n) c_{k}^{*}\right\}=E\left\{x(n) y_{k}^{*}(0)\right\}=R_{x y_{k}}(n)$.

But the cross-power spectrum of $x(n)$ and $y_{k}(n)$ equals $S_{x}(\omega) H_{k}^{*}(\omega)$, therefore

$$
\begin{aligned}
E\left\{x(n) c_{k}^{*}\right\} & =\frac{1}{2 \pi} \int_{\pi}^{\pi} S_{x}(\omega) H_{k}^{*}(\omega) \exp (j \omega n) d \omega \\
& =\frac{1}{2 \pi} \int_{(k-1 / 2) \omega_{0}}^{(k+1 / 2) \omega_{0}} S_{x}(\omega) \exp (j \omega n) d \omega .
\end{aligned}
$$

Summing along all coefficients, we get

$$
\begin{aligned}
& \sum_{k=0}^{L-1} E\left\{x(n) c_{k}^{*}\right\} \exp \left(-j k \omega_{0} n\right) \\
& \quad=\frac{1}{2 \pi} \sum_{k=0}^{L-1} \int_{(k-1 / 2) \omega_{0}}^{(k+1 / 2) \omega_{0}} S_{x}(\omega) \exp \left[j\left(\omega-k \omega_{0}\right) n\right] d \omega .
\end{aligned}
$$

Using Equation (4), the left-hand side of the previous equation simplifies to

$$
\begin{aligned}
E\left\{x(n) \hat{x}^{*}(n)\right\}= & \frac{1}{2 \pi} \sum_{k=0}^{L-1} \int_{(k-1 / 2) \omega_{0}}^{(k+1 / 2) \omega_{0}} S_{x}(\omega) \\
& \times \exp \left[j\left(\omega-k \omega_{0}\right) n\right] d \omega \\
= & \frac{1}{2 \pi} \sum_{k=0}^{L-1} \int_{(k-1 / 2) \omega_{0}}^{(k+1 / 2) \omega_{0}} S_{x}(\omega) \\
& \times\left[\cos \left(\omega-k \omega_{0}\right) n\right. \\
& \left.+j \sin \left(\omega-k \omega_{0}\right) n\right] d \omega .
\end{aligned}
$$

Since $S_{x}(\omega)$ is an even function, the second term in the previous equation equates to zero, hence

$$
\begin{aligned}
E\left\{x(n) \hat{x}^{*}(n)\right\}= & \frac{1}{2 \pi} \sum_{k=0}^{L-1} \int_{(k-1 / 2) \omega_{0}}^{(k+1 / 2) \omega_{0}} S_{x}(\omega) \\
& \times \cos \left[\left(\omega-k \omega_{0}\right) n\right] d \omega .
\end{aligned}
$$

Substituting this equation and the result from Equation (26) in Equation (34), we get

$e_{m s}=\frac{1}{\pi} \sum_{k=0}^{L-1} \int_{(k-1 / 2) \omega_{0}}^{(k+1 / 2) \omega_{0}} S_{x}(\omega)\left[1-\cos \left(\omega-k \omega_{0}\right) n\right] d \omega$.

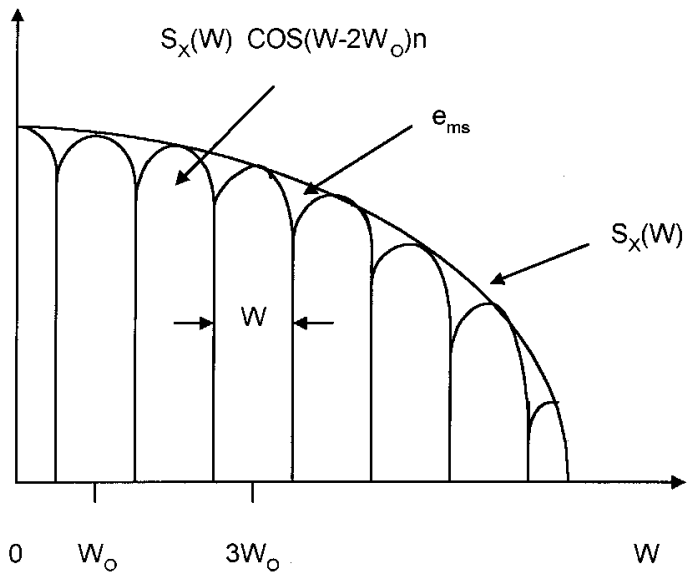

Fig. 3 Mean square error of an arbitrary low-pass signal.

For an arbitrary low pass signal, this equation is plotted as a shaded area in Fig. 3. The second part of this equation depends on $n$ and is generated by $E\left\{\left|x(n) \hat{x}^{*}(n)\right|\right\}$. This shows that the $x(n)$ and $\hat{x}(n)$ are individually but not jointly wide-sense stationary. If they were, the mean square error $e_{m s}$ would be independent of time.

The prior is a worst case estimate assuming that $S_{x}(\omega)$ is concentrated at the end points of each integration interval, i.e., from $(k-1 / 2) \omega_{0}$ to $(k+1 / 2) \omega_{0}$. If $S_{x}(\omega)$ does not vary appreciably in these intervals, then Equation (17) can be modified by replacing $S_{x}(\omega)$ by a constant in each integration interval as

$$
\begin{aligned}
e_{m s} & =\frac{1}{\pi} \sum_{k=0}^{L-1} S_{x}(\omega)\left[\omega_{0}-\frac{\sin \left(\omega_{0} n / 2\right)}{n}-\frac{\sin \left(\omega_{0} n / 2\right)}{n}\right] \\
& =\frac{1}{\pi} \sum_{k=0}^{L-1} S_{x}(\omega) \omega_{0}\left[1-\frac{\sin \left(\omega_{0} n / 2\right)}{\omega_{0} n / 2}\right], \\
e_{m s} & =2\left[1-\frac{\sin \left(\omega_{0} n / 2\right)}{\omega_{0} n / 2}\right] \frac{1}{2 \pi} \sum_{k=0}^{L-1} \omega_{0} S_{x}(\omega) .
\end{aligned}
$$

Since $S_{x}(\omega)$ does not vary appreciably in each integration interval, then using property $(e)$, the previous equation can be written as

$e_{m s}=2\left[1-\frac{\sin \left(\omega_{0} n / 2\right)}{\omega_{0} n / 2}\right] \sum_{k=0}^{L-1} E\left\{\left|c_{k}\right|^{2}\right\}$.

As proven in the property in Sec. 3.6, this can also be simplified as

$\epsilon_{m s} \approx 2 E\left\{|x(n)|^{2}\right\}\left[1-\frac{\sin \left(\frac{\omega_{0} n}{2}\right)}{\frac{\omega_{0} n}{2}}\right]$.

This means that in the limit as $\omega_{0}$ approaches zero, the mean square error approaches zero in a mean square sense. We can also deduce from Equation (44) that for the mean square error not to exceed a certain percentage of average 
Table 1 Real component of AFE basis functions.

\begin{tabular}{lllcrrrr}
\hline \hline 0.0892 & 0.1059 & 0.1179 & 0.1242 & 0.1242 & 0.1179 & 0.1059 & 0.0892 \\
0.0892 & 0.0749 & 0 & -0.0878 & -0.1242 & -0.0834 & 0 & 0.0631 \\
0.0892 & 0 & -0.1179 & 0 & 0.1242 & 0 & -0.1059 & 0 \\
0.0892 & -0.0749 & 0 & 0.0878 & -0.1242 & 0.0834 & 0 \\
0.0892 & -0.1059 & 0.1179 & -0.1242 & 0.1242 & -0.1179 & 0.1059 & -0.0892 \\
0.0892 & -0.0749 & 0 & 0.0878 & -0.1242 & 0.0834 & 0 & -0.0631 \\
0.0892 & 0 & -0.1179 & 0 & 0.1242 & 0 & -0.1059 \\
0.0892 & 0.0749 & 0 & -0.0878 & -0.1242 & -0.0834 & 0 & 0 \\
\hline \hline
\end{tabular}

power of $x(n)$, the first null of sinc function in Equation (44) shall fall very far away from the origin. Since first null of the sinc function in an ACE falls at $2 L$ instead of $L$ as in an AFE, mean square error introduced by ACE will be less than the error due to applying AFE on the signal.

\section{Determination of Transform Efficiency}

In this section, we determine the efficiency of the expansions by using the first order Markov process. This model represents the gross behavior of image sources moderately well, even though it fails if accurate modeling is required. Specifically, we compute energy packing efficiency and the variance of the coefficients. To demonstrate the approach, we take first order Markov model as ${ }^{5}$ :

$\psi=\left[\begin{array}{cccccc}1 & \rho & \rho^{2} & \cdot & \cdot & \rho^{N-1} \\ \rho & 1 & \rho & \cdot & \cdot & \rho^{N-2} \\ \cdot & & & & & \cdot \\ \cdot & & & & & \cdot \\ \cdot & & & & & \cdot \\ \rho^{N-1} & \rho^{N-2} & . & . & . & 1\end{array}\right], \quad 0<\rho<1$,

where $\psi$ provides a useful model for the data covariance matrix corresponding to the rows and columns of an image matrix and $\rho$ is the interelement correlation coefficient. The covariance matrix in the transform domain is denoted by $\psi$ and is given by ${ }^{6}$

$\Psi=\Lambda \psi \Lambda * T$,

where $\Lambda$ is the two-dimensional matrix representation of a transform and $\Lambda^{*}$ is its complex conjugate. Since AFE and ACE expansions have nonorthogonal basis functions and DFT and discrete cosine transform (DCT) are orthogonal transforms, we compare transform efficiency using an equal number of transform coefficients i.e., $L=N$. The basis functions of AFE and ACE for $N=8$ and $L=8$ are computed from the following equations [see Equation (3) and (29)]

$$
\begin{aligned}
\text { AFE: } c_{k, n} & =\frac{\sin \left(\frac{n \pi}{L}\right)}{n \pi} \exp \left(-j \frac{2 \pi k n}{L}\right) \\
\text { ACE: } c_{k, n} & =\frac{\sin \left(\frac{n \pi}{2 L}\right)}{n \pi} \cos \left[\frac{\pi k(2 n+1)}{2 L}\right] .
\end{aligned}
$$

The computed basis functions are shown in Tables 1 through 3 . We examine the transform efficiency, decorrelation efficiency, and the variance of coefficients of ACE, AFE, DFT, and DCT for a relatively higher value of interelement correlation and smaller block size, because compression of the signals is useful only when signals are highly correlated, and most of the common image coding algorithms use block sizes of $8 \times 8$ or $16 \times 16$. We determine the transform efficiency of AFE, ACE, DFT, and DCT by examining the diagonal elements of their respective transform domain covariance Equation (46) for $\rho$ $=0.91, N=16$, and $L=16$, where $\Lambda$ represents the respective transformation matrix. The relative amount of energy in the first $M$ of the total $N$ diagonal components is given as $^{6}$

$\eta_{\text {transform }}=\frac{\sum_{j, k=1}^{M} Y_{j, k}}{\sum_{j, k=1}^{N} X_{j, k}}, \quad j=k$,

where $\Sigma X_{j, k}$ is the total sum of data covariance entries and $\sum Y_{j, k}$ is the total sum of transform covariances. We calculated the energy packing efficiency of DCT, DFT, ACE, and AFE by looking at diagonal elements of their transform covariance matrices when $j=k$, and $M$ varies from 1 to $N$.

Table 2 Imaginary component of AFE basis functions.

\begin{tabular}{llllllll}
\hline \hline 0 & 0 & 0 & 0 & 0 & 0 & 0 & 0 \\
0 & $-0.0749 j$ & $-0.1179 j$ & $-0.0878 j$ & 0 & $0.0834 j$ & $0.1059 j$ & $0.0631 j$ \\
0 & $-0.1059 j$ & 0 & $0.1242 j$ & 0 & $-0.1179 j$ & $0.0892 j$ \\
0 & $-0.0749 j$ & $0.1179 j$ & $-0.0878 j$ & 0 & $0.0834 j$ & $-0.1059 j$ & $0.0631 j$ \\
0 & 0 & 0 & 0 & 0 & 0 & 0 & $0.1059 j$ \\
0 & $0.0749 j$ & $-0.1179 j$ & $0.0878 j$ & 0 & $-0.0834 j$ & $-0.0631 j$ \\
0 & $0.1059 j$ & 0 & $-0.1242 j$ & 0 & $0.1179 j$ & $-0.0892 j$ \\
0 & $0.0749 j$ & $0.1179 j$ & $0.0878 j$ & 0 & $-0.0834 j$ & $-0.1059 j$ & $-0.0631 j$ \\
\hline \hline
\end{tabular}


Table 3 ACE basis functions.

\begin{tabular}{lllrrrrr}
\hline \hline 0.1154 & 0.12 & 0.1232 & 0.1248 & 0.1248 & 0.1232 & 0.12 & 0.1154 \\
0.1132 & 0.0998 & 0.0684 & 0.0243 & -0.0243 & -0.0684 & -0.0998 & -0.1132 \\
0.1066 & 0.0459 & -0.0471 & -0.1153 & -0.1153 & -0.0471 & 0.0459 & 0.1066 \\
0.0959 & -0.0234 & -0.1208 & -0.0693 & 0.0693 & 0.1208 & 0.0234 & -0.0959 \\
0.0816 & -0.0849 & -0.0871 & 0.0882 & 0.0882 & -0.0871 & -0.0849 & 0.0816 \\
0.0641 & -0.1177 & 0.024 & 0.1038 & -0.1083 & -0.024 & 0.1177 \\
0.0442 & -0.1109 & 0.1138 & -0.0478 & -0.0478 & 0.1138 & -0.1109 & 0.0641 \\
0.0225 & -0.0667 & 0.1024 & -0.1224 & 0.1224 & -0.1024 & 0.0667 & -0.0225 \\
\hline \hline
\end{tabular}

Results are shown in Fig. 4. The best performance is given by ACE followed closely by DCT and AFE. The larger values of $\eta$, even for relatively small values of $M$, are characteristics of all transforms and are the result of high interelement correlation within the data. Good energy packing efficiency also demands that the magnitudes of the variances fall off rapidly with increasing coefficient order. To compare transform coefficient variances of DCT, DFT, and approximate trigonometric expansions, we again compared the diagonal elements of their respective transform domain covariance matrices. The diagonal elements of each transform domain covariance matrix are shown in Fig. 5, which shows that the performance of ACE is the best followed by DCT and AFE. Owing to the symmetry inherent within the basis matrix, the AFE and DFT have pairs of coefficients of equal variance, and this accounts for the step-like trend of their curves. To demonstrate decorrelation efficiency of trigonometric expansions, we use the same first order Markov model by calculating the decrease in interelement correlation in the transform domain covariance matrix compared with that in the data domain equivalent. The decorrelation efficiency is then given ${ }^{6}$ as

$\eta_{\text {decorrelation }}=1-\frac{\sum_{j, k=1}^{N} Y_{j, k}}{\sum_{j, k=1}^{N} X_{j, k}}, \quad j \neq k$,

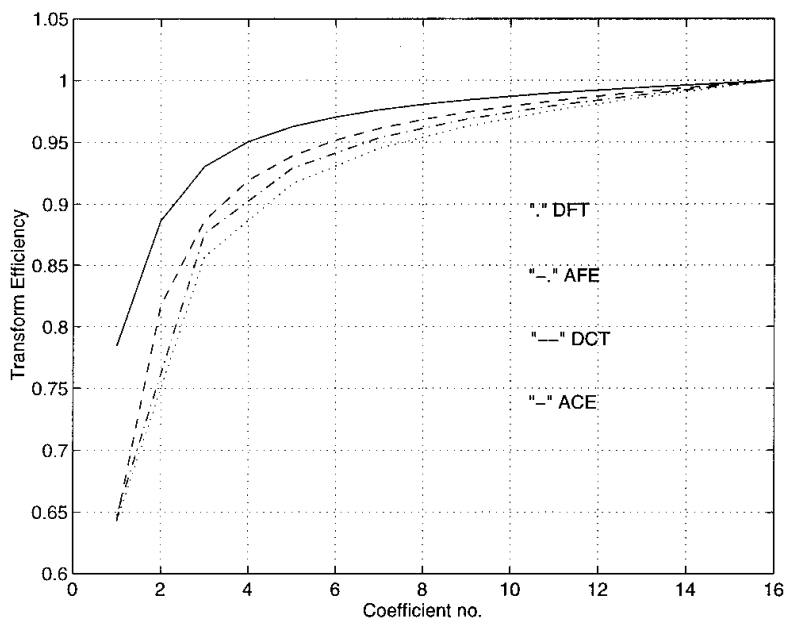

Fig. 4 Energy packing efficiency versus coefficient order. where $\sum X_{j, k}$ and $\sum Y_{j, k}$ are defined as before. We computed the decorrelation efficiency of approximate trigonometric expansions, discrete cosine, and Fourier transforms for various values of $N$ and $\rho$, and the results are shown in Table 4. It is clear from Table 4 that approximate trigonometric expansions provide better interelement decorrelation than discrete cosine and Fourier transforms.

\section{Implementation and Experimental Results}

In this section we present experimental results. The performance of the approximate trigonometric expansions is evaluated by using an objective fidelity criterion. One commonly used objective fidelity criterion is the mean square signal to noise ratio $\left(\mathrm{SNR}_{\mathrm{ms}}\right)$, defined as:

$\mathrm{SNR}_{\mathrm{ms}}=\frac{\sum_{i=0}^{N-1} \sum_{j=0}^{N-1}\left(\hat{x}_{i j}\right)^{2}}{\sum_{i=0}^{N-1} \sum_{j=0}^{N-1}\left(x_{i, j}-\hat{x}_{i, j}\right)^{2}}$,

where $\hat{x}(i, j)$ and $x(i, j)$ are reconstructed and original image pixels, respectively. Since the performance of the expansions depends on the choice of $\omega_{0}=2 \pi / L$, the expansions were applied to the "Lena' image, and the $\mathrm{SNR}_{\mathrm{ms}}$ was computed for various values of $L$. Results are shown in Fig. 6, where Fig. 6(a) shows the original "Lena' image and Figs. 6(b), 6(c), 6(d), and 6(e) show reconstructed

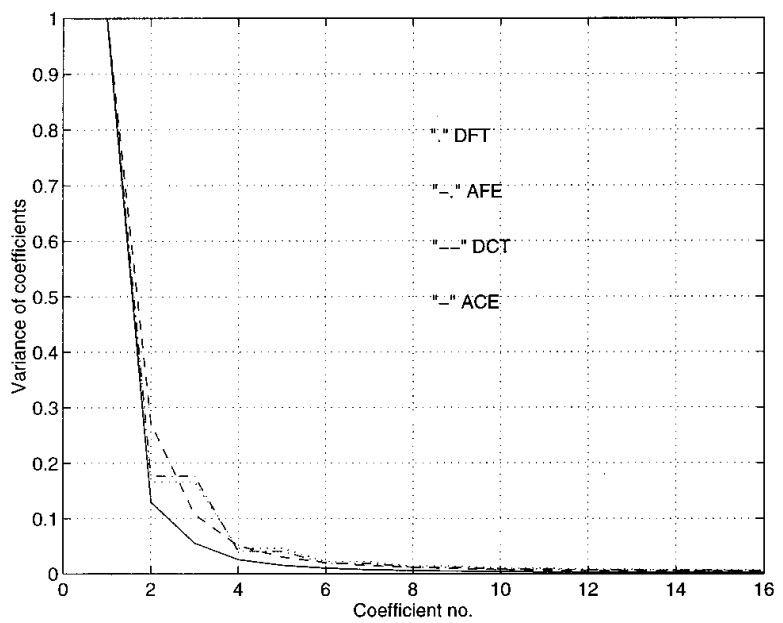

Fig. 5 Variances of coefficients versus coefficient order. 
Table 4 Decorrelation efficiency of trigonometric expansions and DFT/DCT for various values of $N$ and $\rho$.

\begin{tabular}{|c|c|c|c|c|c|c|c|c|c|c|c|c|}
\hline \multirow[b]{2}{*}{$\rho$} & \multicolumn{4}{|c|}{$N=8$} & \multicolumn{4}{|c|}{$N=16$} & \multicolumn{4}{|c|}{$N=32$} \\
\hline & 0.85 & 0.9 & 0.95 & 0.98 & 0.85 & 0.9 & 0.95 & 0.98 & 0.8 & 0.9 & 0.95 & 0.98 \\
\hline DFT & 0.831 & 0.883 & 0.94 & 0.975 & 0.782 & 0.839 & 0.911 & 0.962 & 0.772 & 0.816 & 0.886 & 0.948 \\
\hline DCT & 0.966 & 0.978 & 0.989 & 0.995 & 0.963 & 0.976 & 0.988 & 0.995 & 0.962 & 0.975 & 0.988 & 0.995 \\
\hline AFE & 0.982 & 0.986 & 0.99 & 0.992 & 0.991 & 0.993 & 0.995 & 0.997 & 0.996 & 0.996 & 0.997 & 0.998 \\
\hline ACE & 0.996 & 0.997 & 0.998 & 0.998 & 0.998 & 0.998 & 0.999 & 0.999 & 0.999 & 0.999 & 0.999 & 0.999 \\
\hline
\end{tabular}

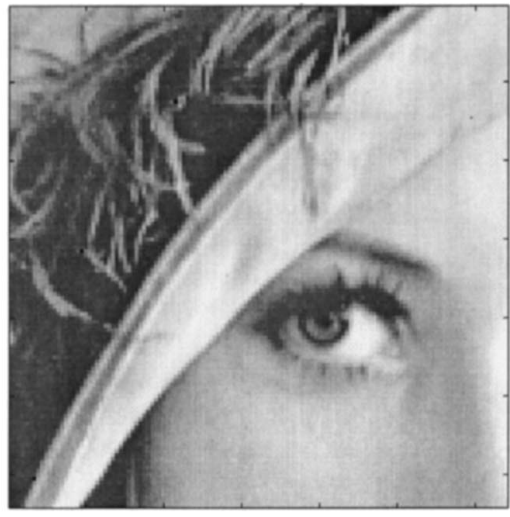

(a)

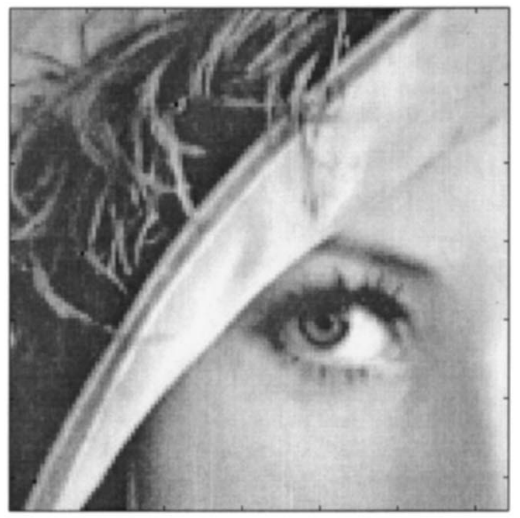

(c)

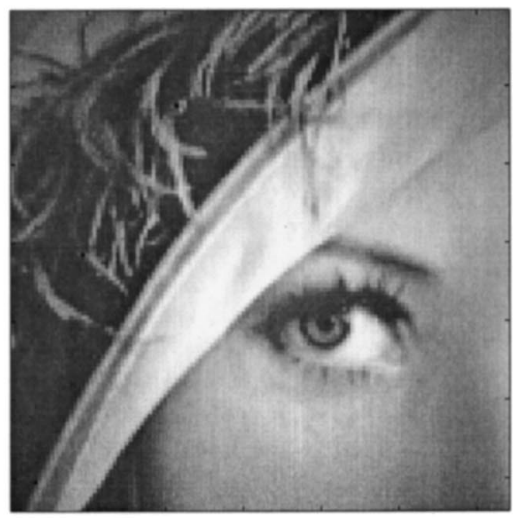

(b)

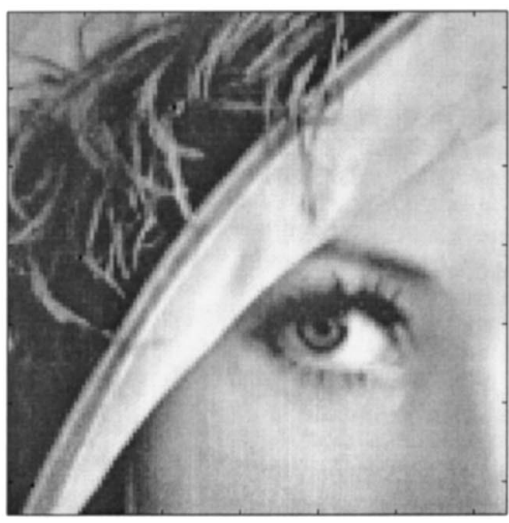

(d)

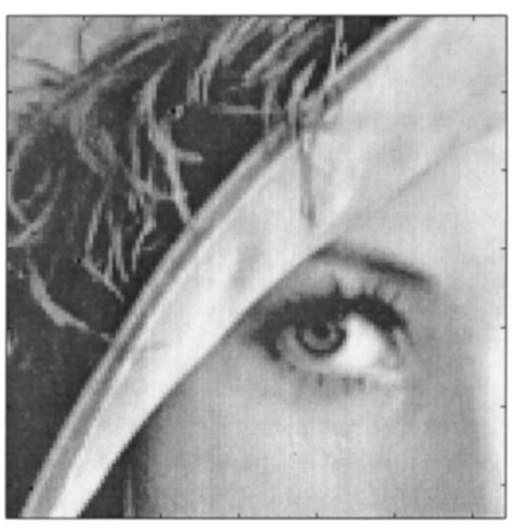

(e)

Fig. 6 (a) Original "Lena" image; (b) image reconstructed with AFE $(L=N), S R_{m s}=26 \mathrm{~dB}$; (c) image reconstructed with $\operatorname{AFE}(L=2 N), \mathrm{SNR}_{\mathrm{ms}}=50.9 \mathrm{~dB}$; (d) image reconstructed with $\operatorname{ACE}(L=2 N)$, $\mathrm{SNR}_{\mathrm{ms}}=50.6 \mathrm{~dB}$; and $(\mathrm{e})$ image reconstructed with $\mathrm{ACE}(L=2 N), \mathrm{SNR}_{\mathrm{ms}}=53.9 \mathrm{~dB}$. 


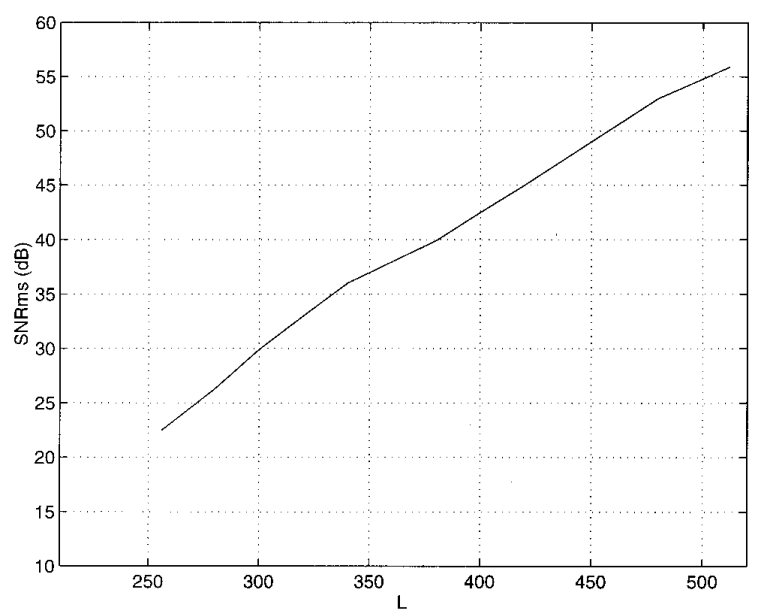

Fig. $7 \mathrm{SNR}_{\mathrm{ms}}$ of reconstructed "Lena" image versus $L$.

"Lena" images using AFE and ACE, respectively, for two different values of $L$. It is clear from the figures that for the same $L$, the $\mathrm{SNR}_{\mathrm{ms}}$ due to ACE is higher than AFE. This verifies our results in Sec. 4. This is expected, since a higher value of $L$ results in the computation of a relatively larger number of coefficients yielding less error in the approximation of the signal spectrum, whereas smaller values of $L$ produce a coarse resolution resulting in a large error. This is in contrast to the discrete cosine and Fourier transforms, where the frequency resolution is determined by the size of the data vector. Figure 7 shows $\mathrm{SNR}_{\mathrm{ms}}$ of the reconstructed "Lena" image when $L$ varies from low to high. It is clear from Fig. 7 that larger values of $L$ (and hence more coefficients to be computed) yield higher $\mathrm{SNR}_{\mathrm{ms}}$. To test the performance of the approximate trigonometric expansions for coding of images, thresholding was applied to $\mathrm{ACE}$ and AFE coefficients. For comparison purposes, thresholding was also applied on the DCT coefficients of the "Lena" image. Three different thresholds, each for DCT, AFE, and ACE coefficients, were used to retain an equal number of coefficients. The number of retained coefficients was $13 \%$ of the total coefficients for all of the three cases. Results are shown in Figs. 8(a) through 8(c) Poor $\mathrm{SNR}_{\mathrm{ms}}$ of Fig. 8(a) accounts mainly for the windowing effect present in the reconstructed image. It is clear from the figures that ACE has better performance than DCT and AFE. This verifies our results in Sec. 6.

\section{Conclusions and Future Work}

We have presented an analysis of approximate trigonometric expansions of sampled signals along with simulation results. It was shown that for first order Markov processes, the transform efficiency of the ACE is better than the DCT and the AFE. ACE was also tested on the "Lena" image for coding purposes and the simulations showed that its performance is better than DCT and AFE. It was noted that the resolution of AFE and ACE coefficients is user defined and depends on $L$. Since practical transform coding of images is performed using blocks of images, $L$ can be used as an additional parameter for adaptive coding of images. Ad-

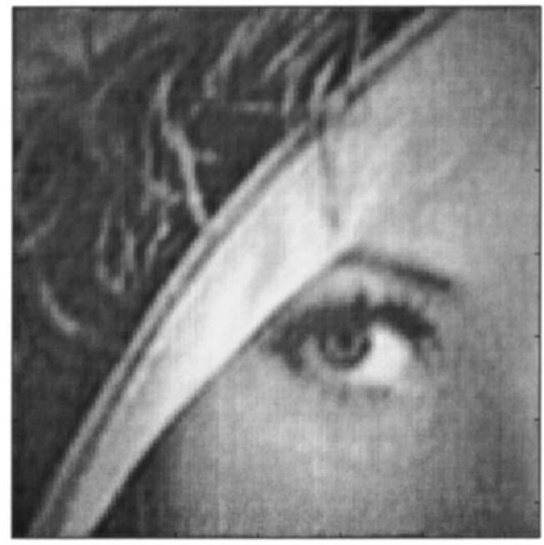

(a)

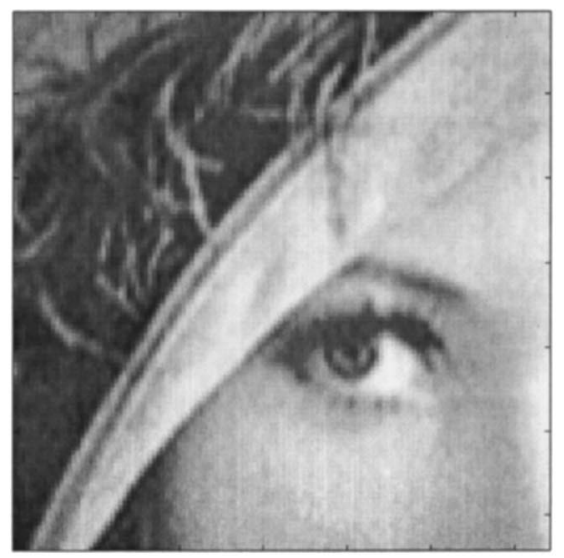

(b)

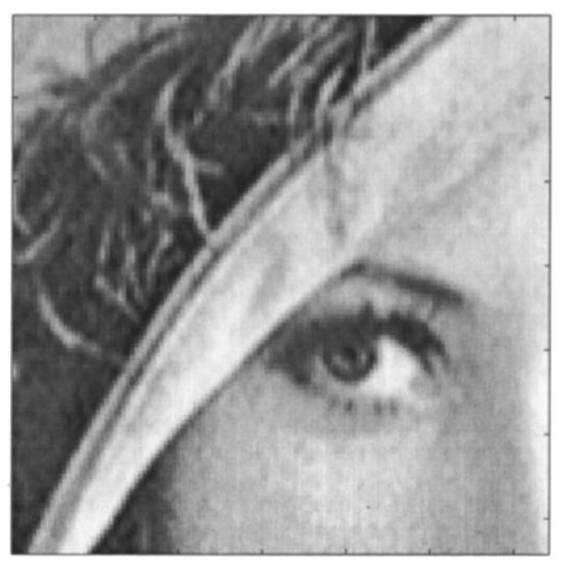

(c)

Fig. 8 (a) Image reconstructed with AFE $(L=N), \mathrm{SNR}_{\mathrm{ms}}=13 \mathrm{~dB}$; (b) image reconstructed with $\mathrm{DCT} S \mathrm{SNR}_{\mathrm{ms}}=32 \mathrm{~dB}$; and (c) image reconstructed with ACE $(L=N), S_{N} R_{m s}=34 \mathrm{~dB}$.

ditionally, this characteristic can also be used for multiresolution analysis of the signal. Our future tasks will be to explore these possibilities.

\section{References}

1. V. D. Vaughn and T. S. Wilkinson, "System considerations for multispectral image compression designs," IEEE Sig. Process. Mag., pp. 19-31 (January 1995). 
2. A. K. Jain, "Image data compression: a review," Proc. IEEE 69(3), 349-389 (March 1991).

3. A. K. Jain, "A sinusoidal family of unitary transforms," IEEE Trans. Patt. Anal. Mach. Intell. PAMI-1, 356-365 (Oct. 1979).

4. A. Papoulis, "Probability, Random variables, and Stochastic Processes," McGraw-Hill Book Company, New York (1965).

5. N. Ahmed, T. Natarajan, and K. R. Rao, "Discrete cosine transform," IEEE Trans. Comput. C-23, 90-93 (1974).

6. R. J. Clarke, "Transform Coding of Images," Academic Press Inc., London Ltd. (1985).

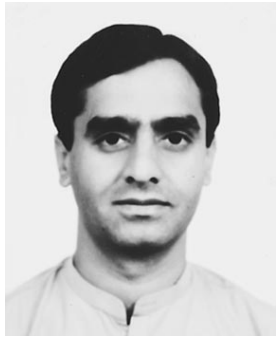

Qurban A. Memon received his BE degree in electronic engineering from $\mathrm{Me}$ hran University of Engineering and Technology, Jamshoro, Pakistan; the ME degree in electrical engineering from the University of Florida, Gainesville, in 1993 and the PhD degree in electrical engineering from the University in Central Florida Orlando, in 1996. In January 1997 he joined the Ghulam Ishaq Khan Institute of Engineering Sciences and Technology,

Pakistan, where he is currently an assistant professor in the Depart- ment of Electronic Engineering. His research interests are in digital signal and image processing, image compression, and DSP solutions for mobile and cellular communications.

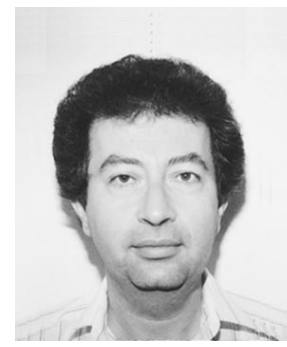

Takis Kasparis received the diploma of electrical engineering from the National Technical University of Athens, Greece in 1980 , and the MEEE and $\mathrm{PhD}$ degrees in electrical engineering from the City College of New York in 1982 and 1988. From 1985 until 1989 he was an electronics consultant. In 1989 he joined the electrical engineering department of the University of Central Florida, Orlando, where he is presently an associate professor. His research interests are in digital signal and image processing, texture analysis, and computer vision. 